\title{
CHRONIC IMPAIRMENT OF AXONAL TRANSPORT ELIMINATES TASTE RESPONSES AND TASTE BUDS ${ }^{1}$
}

\author{
HARRY E. SLOAN, STEPHEN E. HUGHES, AND BRUCE OAKLEY ${ }^{2}$ \\ Division of Biological Sciences, The University of Michigan, Ann Arbor, Michigan 48109
}

Received April 5, 1982; Revised June 25, 1982; Accepted July 12, 1982

\begin{abstract}
A Silastic nerve cuff containing colchicine $(1 \% \mathrm{w} / \mathrm{v})$ was placed around the combined lingualchorda tympani nerve of the Mongolian gerbil (Meriones unguiculatus) to evaluate the role of axonal transport in the maintenance of taste buds. After 3 days the summated gustatory impulse discharges recorded from the chorda tympani nerve were reduced by $60 \%$, while compound action potentials had not changed appreciably. The lingual-chorda tympani nerve underwent ultrastructural changes including a loss of microtubules, an increased prominence and disorientation of neurofilaments, and a significant shrinkage in the cross-sectional area of axoplasm. The shrinkage of axoplasm and the accumulation of mitochondria and cholinesterase at the nerve cuff provided evidence that the colchicine treatment acted to impair axonal transport. More substantial pathological changes were evident in nerve ultrastructure by 15 days when both the ipsilateral chorda tympani taste responses and fungiform taste buds were nearly absent. Control cuffs lacking colchicine had little effect on chorda tympani taste responses, taste buds, or nerve ultrastructure. Eight or 15 days of nerve exposure to lumicolchicine, an isomer of colchicine with low affinity for tubulin, had no significant effect on taste responses. $\left[{ }^{3} \mathrm{H}\right]$ Colchicine was used in the nerve cuff to demonstrate that colchicine must have acted directly upon the nerve trunk, rather than the taste buds, to cause the loss of taste responses and taste buds. $\left[{ }^{3} \mathrm{H}\right]$ Colchicine levels were equal in the two sides of the tongue, whereas both the functional and structural deterioration of the taste buds were restricted to the ipsilateral side. We conclude that the loss of taste responses and taste buds was caused by chronically impaired axonal transport in gustatory axons.
\end{abstract}

The evidence presently available suggests that the structure and function of mammalian taste buds are maintained by trophic influences of gustatory nerves. The classic observations are that denervation causes taste buds to degenerate and subsequent reinnervation of the gustatory epithelium causes taste buds to re-form (e.g., Vintschgau and Hönigschmied, 1877; Guth, 1957; Fujimoto and Murray, 1970; Cheal and Oakley, 1977). One model explains these observations by the continuous axonal transport of neurotrophic chemicals along gustatory axons. This model predicts the recent observations that both the rate of loss of gustatory discharges (Oakley et al., 1980) and the rate of loss of taste buds (State and Dessouky, 1977) depend upon the length of the transected nerve remaining attached to the tongue; taste buds with shorter nerve stumps deteriorate more rapidly.

\footnotetext{
'This work was supported in part by National Institutes of Health Grant NS-07072. We are grateful for the assistance of Lee B. Jones, Jayne Minier, Rebecca M. Sloan, Todd Space, and Victoria Vasek.

${ }^{2}$ To whom correspondence should be addressed at Neuroscience Laboratory Building, The University of Michigan, Ann Arbor, MI 48109 .
}

Oakley et al. (1981) have demonstrated that local treatment of a gustatory nerve (IXth), either with colchicine or by cooling, impairs axonal transport and simultaneously reduces taste responses significantly. The operative procedures used in these earlier acute electrophysiological experiments precluded chronic maintenance of the animals. Therefore, it was impossible to determine whether taste bud degeneration also would have occurred since taste buds require several days to degenerate following insult to the gustatory nerve. In the present study we limited the spread of colchicine by incorporating it into a small Silastic cuff chronically implanted around the lingual-chorda tympani nerve of gerbils.

In previous chronic experiments colchicine and vincristine (a Vinca alkaloid which, like colchicine, disrupts both axonal transport and mitosis) have been used to produce taste bud degeneration by the methods of: $(i)$ intraperitoneal injection of colchicine (Beidler and Smallman, 1965) or vincristine (State et al., 1977), (ii) injection of colchicine into the vallate papilla of the rat tongue followed by a second colchicine injection 5 days later (Rodrigo Angulo et al., 1978), and (iii) by soaking the IXth nerve in colchicine (Chelyshev et al., 1981; 
Donegani and Filogamo, 1971). Only Donegani and Filogamo (1971) measured the amount of colchicine reaching the tongue. They found that it initially was 2 to 8 times more concentrated in the ipsilateral side of the tongue.

Chemicals capable of blocking axonal transport might directly arrest the mitosis of taste receptor cells since cells in the taste bud are known to turn over with a halflife of 9 to 10 days (Beidler and Smallman, 1965; Conger and Wells, 1969; Farbman, 1980). Hence, if one is to use transport blocking agents to implicate axonal transport in the maintenance of taste buds, it is essential to rule out direct toxic effects of these chemicals upon cells in the taste bud. The present experiments suggest that colchicine released from Silastic nerve cuffs caused taste bud degeneration by its effect upon axonal transport.

\section{Materials and Methods}

Ninety-six Mongolian gerbils (Meriones unguiculatus) 5 to 9 months old (48.0 to $105.8 \mathrm{gm}$ body weight) were used in this study. Gerbils were maintained on a daily cycle of $12 \mathrm{hr}$ light and $12 \mathrm{hr}$ dark with free access to food and water.

Silastic nerve cuffs. To make the nerve cuffs, colchicine or lumicolchicine was dissolved in absolute ethanol and added to a stainless steel well. A control cuff involved the use of ethanol alone. The well was placed into the dark and the ethanol was allowed to evaporate. Enough Silastic (Medical Adhesive Type A, Dow Corning Corp.) was added to the well with the colchicine to make a $1 \%$ colchicine concentration $(\mathrm{w} / \mathrm{v})$. The mixture then was pressed into an aluminum mold with a volume of $3.8 \mu \mathrm{l}$. A No. 1 insect pin was inserted to create the hollow core of the cuff. After a minimum of $24 \mathrm{hr}$ of curing, the cuff was removed from the mold and trimmed to $1.5 \mathrm{~mm}$ in length with a $1.2-\mathrm{mm}$ outer diameter and a $0.4-\mathrm{mm}$ inner diameter for a final volume of $1.5 \mu$ l. A longitudinal slit in the wall allowed the cuff to be opened for placement around the nerve. A $1 \%$ colchicine cuff contained $15.2 \mu \mathrm{g}$ of colchicine.

Four types of Silastic nerve cuffs were used on the lingual-chorda tympani nerve in this study, with control cuffs lacking colchicine and experimental cuffs containing $1 \%$ colchicine ( $1 \%$ w/v; Sigma Chemical Co. or Aldrich), $1 \%$ colchicine with $3.5 \mu \mathrm{Ci}$ of $\left[{ }^{3} \mathrm{H}\right]$ colchicine (New England Nuclear, 5.0 or $10.1 \mathrm{Ci} / \mathrm{mm}$ ), or $1 \%$ lumicolchicine. Lumicolchicine was prepared by long wave ultraviolet irradiation of colchicine in the manner described by Wilson and Friedkin (1966).

Operative procedures. To implant the cuffs, gerbils were anesthetized with ketamine hydrochloride $(330 \mathrm{mg} /$ $\mathrm{kg}$ of body weight, i.m.). The gerbil was secured in a head holder and the lingual chorda tympani nerves were exposed in the region of the external pterygoid muscle. The Silastic cuff was spread apart and gently placed around the combined lingual and chorda tympani nerves near their junction. Following surgery the animals were housed individually.

Electrophysiological recordings were obtained from the ipsilateral and contralateral chorda tympani nerves proximal to the nerve cuff $1,2,3,4,8$, or 15 days after implantation. Gerbils were anesthetized with ketamine hydrochloride followed $0.5 \mathrm{hr}$ later by sodium pentobarbital $(12.5 \mathrm{mg} / \mathrm{kg}$, i.p.) with additional doses as needed. A rectal thermocouple monitored the gerbil's body temperature, which was maintained at $36^{\circ} \mathrm{C} \pm 2^{\circ} \mathrm{C}$ by a heating pad. The chorda tympani was exposed in the middle ear through the accessory tympanum as described by Jakinovich and Oakley (1975).

Gustatory and electrical stimulation of the lingualchorda tympani nerve. The following taste solutions of reagent grade chemicals were made up in distilled water: $0.5 \mathrm{M}$ sucrose, $0.3 \mathrm{M} \mathrm{NH}_{4} \mathrm{Cl}, 0.3 \mathrm{M} \mathrm{NaCl}, 0.01 \mathrm{~m}$ quinine hydrochloride, and $0.01 \mathrm{~m} \mathrm{HCl}$. For each stimulation a gravity flow system applied 4 to $5 \mathrm{ml}$ to the tongue at a flow rate of $0.3 \mathrm{ml} / \mathrm{sec}$. A 3-min distilled water rinse was alternated with taste solutions without interruption in the continuous flow of fluids. Taste solutions and rinse were presented to the tongue at room temperature $\left(23^{\circ} \mathrm{C}\right.$ $\pm 2^{\circ} \mathrm{C}$ ). Recordings were obtained by touching a $120-\mu \mathrm{m}$ diameter nichrome electrode to the intact chorda tympani where it spans the gap between the incus and the posterior lamina of the tympani bone in the middle ear. An indifferent electrode was placed against the lining of the meatus. The signal was amplified by an AC preamplifier (Grass model P-15) and passed through a 60$\mathrm{Hz}$ filter before being monitored with an oscilloscope and a loud-speaker. The oscilloscope was connected to a magnetic tape recorder (Akai model GX-270D) to provide data storage. Taste responses were summated with an electronic summator (Grass model 7P3A) with the time constant set at $0.5 \mathrm{sec}$. A response was defined as the difference between the steady state level of background activity to flowing distilled water and the peak of the summated discharge elicited by a taste solution.

After taste responses had been recorded in three gerbils with colchicine cuffs implanted for 3 to 4 days, the lingual-chorda tympani nerve of each was electrically stimulated distal and then proximal to the cuff. Stimulation $(0.1$ to $0.6 \mathrm{~mA}, 0.2 \mathrm{msec}, 1.0-\mathrm{Hz}$ biphasic square wave) was provided by a photoelectric stimulus isolation unit and a constant current stimulator (Grass model PSIU6/S8). The compound action potentials, elicited by supramaximal electrical stimulation, were recorded from the chorda tympani in the middle ear.

$\left[{ }^{3} \mathrm{H}\right]$ Colchicine. In 24 animals which received $\left[{ }^{3} \mathrm{H}\right] \mathrm{col}-$ chicine cuffs, the portion of the tongue anterior to the dorsal intermolar eminence was removed at the following times after cuff implantation: $0.25,4,8,24,48$, and 192 $\mathrm{hr}$. The right and left halves were weighed and solubilized separately in $1 \mathrm{ml}$ of Beckman tissue solubilizer $/ 100 \mathrm{mg}$ of wet weight. Aliquots of $100 \mu \mathrm{l}$ were added to scintillation vials containing $10 \mathrm{ml}$ of scintillation cocktail (Beckman Ready-Solv HP) for counting on a Beckman model LSC-230 scintillation counter as described by Oakley et al. (1981). Counts were expressed as counts per minute above background.

Histology. Immediately following electrophysiological recording 3,8 , or 15 days after cuff implantation, tongues were removed and immersed in formalin/sucrose/ $\mathrm{NH}_{4} \mathrm{OH}(10 \% / 15 \% / 1 \%)$. The tongues were embedded in paraffin and sectioned serially at $10 \mu \mathrm{m}$. The sections were stained in Heidenhain's iron hematoxylin to identify fungiform taste buds by the presence of a darkened taste 
pore. The number of fungiform taste buds remaining on the treated side of the tongue was expressed as a percentage of the number on the contralateral side of the tongue.

Histochemistry: Cholinesterase. Gerbils were sacrificed at 18,24, 36, or $48 \mathrm{hr}$ after implantation of colchicine cuffs. The cuff was removed and 5 to $7 \mathrm{~mm}$ of the lingualchorda tympani nerve, including the cuffed portion, were excised and frozen immediately in dry ice. The contralateral nerve served as the control. The nerves were sectioned longitudinally at $16 \mu \mathrm{m}$ in a cryostat. Following fixation in cold buffered formalin, the sections were stained for cholinesterase by a modification of Gomori's stain (Goshgarian, 1977).

Histochemistry: Horseradish Peroxidase. Crystals of horseradish peroxidase (Type II, Sigma Chemical Co.) were placed onto the transected chorda tympani nerve proximal to the junction with the lingual nerve of five gerbils. The incision was closed and the animal was allowed to recover. After 1 to 2 days the combined lingual-chorda tympani nerve was removed and fixed in $4 \%$ glutaraldehyde in phosphate buffer $(\mathrm{pH} 7.4)$. The nerve was embedded in $10 \%$ gelatin, frozen, and sectioned at $40 \mu \mathrm{m}$. Using a modified protocol of Colman et al. (1976), sections were reacted with $o$-dianisidine dihydrochloride (Sigma Chemical Co.).

Electron microscopy. Following electrophysiological recording of taste responses and later removal of the cuff, a segment of the lingual-chorda tympani nerve was removed by transections $2 \mathrm{~mm}$ proximal and $2 \mathrm{~mm}$ distal to the location of the cuff. These nerve segments were taken from a total of 15 colchicine cuff nerves at 3,8 , or 15 days after cuff implantation, from 10 control cuff nerves 3 or 8 days after implantation, and from 15 corresponding segments of contralateral nerves.

The nerves were fixed for $2 \mathrm{hr}$ in $2 \%$ glutaraldehyde in $0.1 \mathrm{M}$ cacodylate buffer $(\mathrm{pH} 7.35)$ and $0.2 \mathrm{M}$ sucrose. After several rinses in the buffer solution the nerves were postfixed for $2 \mathrm{hr}$ in $2 \% \mathrm{OsO}_{4}$, followed by several more rinses. The nerves then were stained in $2 \%$ aqueous uranyl acetate for $1 \mathrm{hr}$, dehydrated in ethanol, and embedded in Spurr's medium (Polysciences, Inc.). Thick and thin cross-sections were cut on a Sorval MT-2 ultramicrotome. Thin sections were stained with lead citrate (Venable and Coggeshall, 1965) and were examined in a Philips EM-300 electron microscope. Analysis of electron micrographs was facilitated by the use of a microcomputer with digitizing tablet (Apple Computers, Inc.), enabling direct measurements of linear features and cross-sectional areas.

\section{Results}

Electrophysiology. The summated taste responses of 48 animals were recorded from the chorda tympani nerve in the middle ear 3,8 , and 15 days after placing a Silastic nerve cuff around the combined lingual-chorda tympani nerve. Experimental nerve cuffs contained either colchicine (with or without $\left[{ }^{3} \mathrm{H}\right]$ colchicine) or lumicolchicine. Control cuffs were made from Silastic without additional chemicals. Taste responses from the untreated contralateral chorda tympani nerve of each animal were indistinguishable from those of normal animals and served as the reference standard against which the effects of colchicine, lumicolchicine, and control cuffs were evaluated. Three days after application of a nerve cuff containing colchicine, the ipsilateral chorda tympani taste responses were reduced more than $60 \%(p<0.002$, Mann-Whitney $U$ test). Median summated taste response magnitudes were severely reduced by 8 days and absent by 15 days; the modal taste response magnitude was $0 \%$ for each chemical at 8 and 15 days. For each time and treatment condition we plot the data from individual animals with the median response magnitude to $0.3 \mathrm{M} \mathrm{NaCl}, 0.5 \mathrm{M}$ sucrose, and $0.3 \mathrm{M} \mathrm{NH}_{4} \mathrm{Cl}$ (Fig. 1). There was no tendency for the response to any one of the five chemicals to be more severely affected. Taste responses obtained from nerves with control cuffs were not significantly different from those obtained from contralateral nerves or from nerves treated with lumicolchicine (Fig. 1).

Taste bud structure. In 40 animals the status of fungiform taste buds was examined by light microscopy 3,8 , or 15 days after applying colchicine or control cuffs to the lingual-chorda tympani nerve. Compared to control cuffs, colchicine-containing cuffs produced a progressive decline in the number of taste buds $(69 \%$ loss at 8 days, $p<0.006 ; 94 \%$ loss at 15 days, $p<0.001$, Mann-Whitney $U$ test). The apparent loss of some fungiform taste buds with the use of control cuffs was not statistically significant. These results are shown in Figure 2. Contralateral fungiform taste buds were normal. Rarely, the colchicine cuff treatment was ineffective; that is, one animal at 8 days and one at 15 days had only minor reductions in taste buds and taste responses.

Mode of action of colchicine. We wished to determine
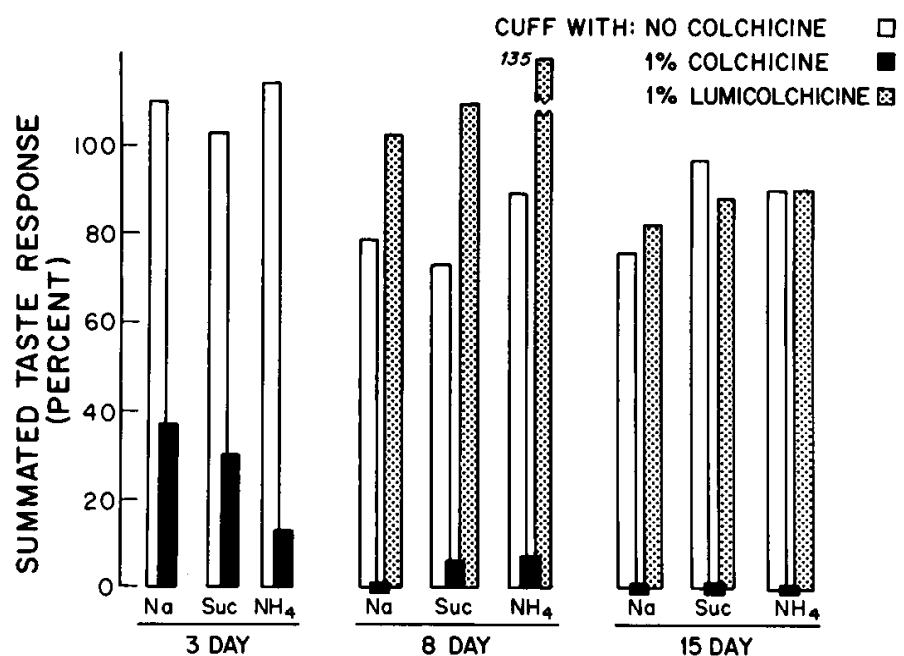

DAYS AFTER NERVE CUFF

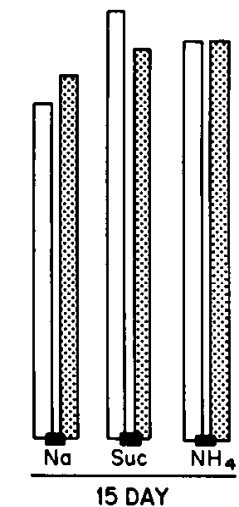

IMPLANTATION

Figure 1. Summated taste responses of the chorda tympani nerve 3,8 , and 15 days after placement around the lingualchorda tympani nerve of the Silastic cuff containing $1 \%$ colchicine (black bars), $1 \%$ lumicolchicine (dotted bars) or no added chemical (open bars). Median values of the peak summated response are given as a percentage of the taste response of the contralateral untreated chorda tympani nerve of the same animal. $\mathrm{Na}, 0.3 \mathrm{M} \mathrm{NaCl}$; Suc, $0.5 \mathrm{M}$ sucrose; $\mathrm{NH}_{4}, 0.3 \mathrm{M} \mathrm{NH}_{4} \mathrm{Cl}$. The bars represent the median response of 5 to 9 animals per treatment condition. 


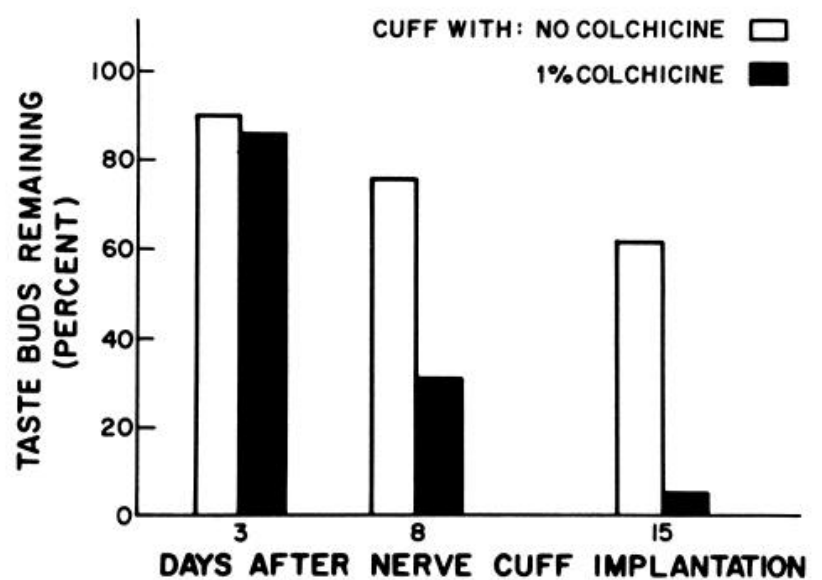

Figure 2. Fungiform taste buds remaining 3, 8, and 15 days after treating the ipsilateral lingual-chorda tympani nerve with a control Silastic nerve cuff or a nerve cuff containing $1 \%$ colchicine. The median number of fungiform taste buds is plotted as a percentage of fungiform taste buds contralateral to the nerve cuff. The tongues of 5 to 8 animals were examined per treatment.

whether the loss of taste bud structure and function might have stemmed from a direct action of colchicine at the level of the taste buds in the tongue. Twenty-four animals were sacrificed at various intervals after implantation of Silastic nerve cuffs containing colchicine and $3.5 \mu \mathrm{Ci}\left[{ }^{3} \mathrm{H}\right]$ colchicine. The amount of colchicine in the tongue peaked at $24 \mathrm{hr}$ at $1.8 \mathrm{ng} / 100 \mathrm{mg}$ of tongue and was at all times equal on the two sides of the tongue (Fig. $3)$. Impaired structure and function of fungiform taste buds were found only ipsilateral to the nerve cuff.

Increased systemic levels of colchicine were produced in six animals by the use of cuffs containing up to 50 times more colchicine. Although these more potent cuffs, implanted around the inferior alveolar nerve, were in close proximity to the lingual-chorda tympani nerve, no change in ipsilateral taste responses was observed.

The effect of $1 \%$ colchicine cuffs on impulse conduction was evaluated in three gerbils whose chorda tympani taste responses had failed after 3 to 4 days. The compound action potential recorded from the chorda tympani in the middle ear was similar whether elicited by electrical stimulation distal or proximal to the nerve cuff. Hence, the impulse mechanisms of the nerve trunk were not blocked by colchicine.

We reasoned that if interference with axonal transport were to be implicated in the decline in the taste responses at 3 days or the loss of taste buds at 8 days, it would be valuable to demonstrate impaired transport prior to these deficits. Accordingly, as a marker of transport, we examined in six gerbils the distribution of cholinesterase in the lingual-chorda tympani nerve which had been treated with a $1 \%$ colchicine nerve cuff for 1 or 2 days. Marked accumulation of cholinesterase was observed proximal to the nerve cuff (Fig. $4 A$ ) with lesser amounts accumulated distally. Contralateral nerves in animals exposed to colchicine for 1 to 3 days showed dispersed background staining (Fig. $4 B$ ).

HRP determination of chorda tympani pathway. We examined the spatial distribution of chorda tympani fibers by applying horseradish peroxidase to the cut end of the chorda tympani nerve. Near the lingual-chorda tympani junction, in the vicinity of the nerve cuff, the chorda tympani axons remain as a small, distinct fascicle on the perimeter of the common nerve.

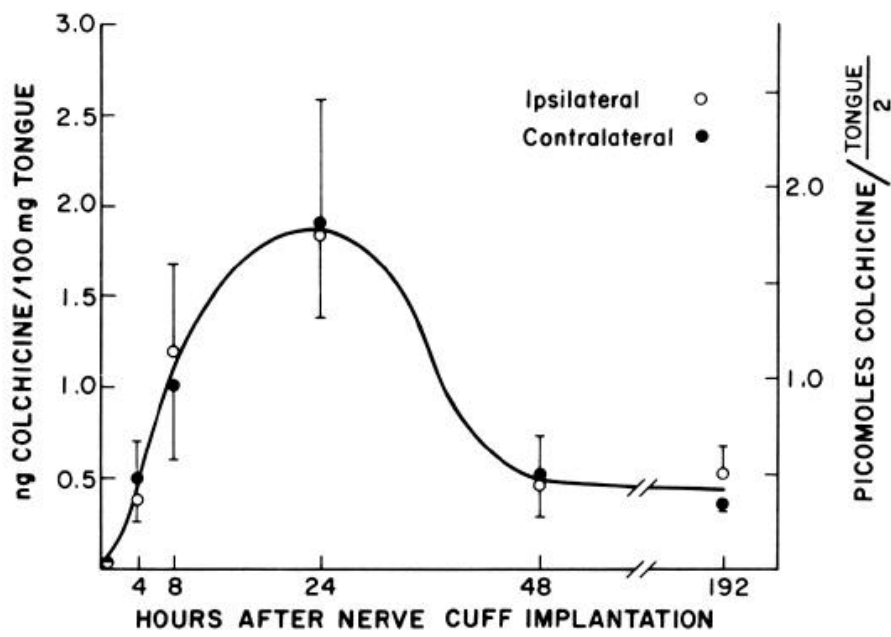

Figure 3. Levels of colchicine in the tongue are shown as a function of time following placement of a $\left[{ }^{3} \mathrm{H}\right]$ colchicine-containing Silastic nerve cuff around the lingual-chorda tympani nerve. Tritium disintegrations were counted for the left (ipsilateral to cuff) and right halves of the tongue anterior to the intermolar eminence. Five animals were used for each time period, except three at $4 \mathrm{hr}$ and one at $15 \mathrm{~min}$. Mean \pm 1 SEM.

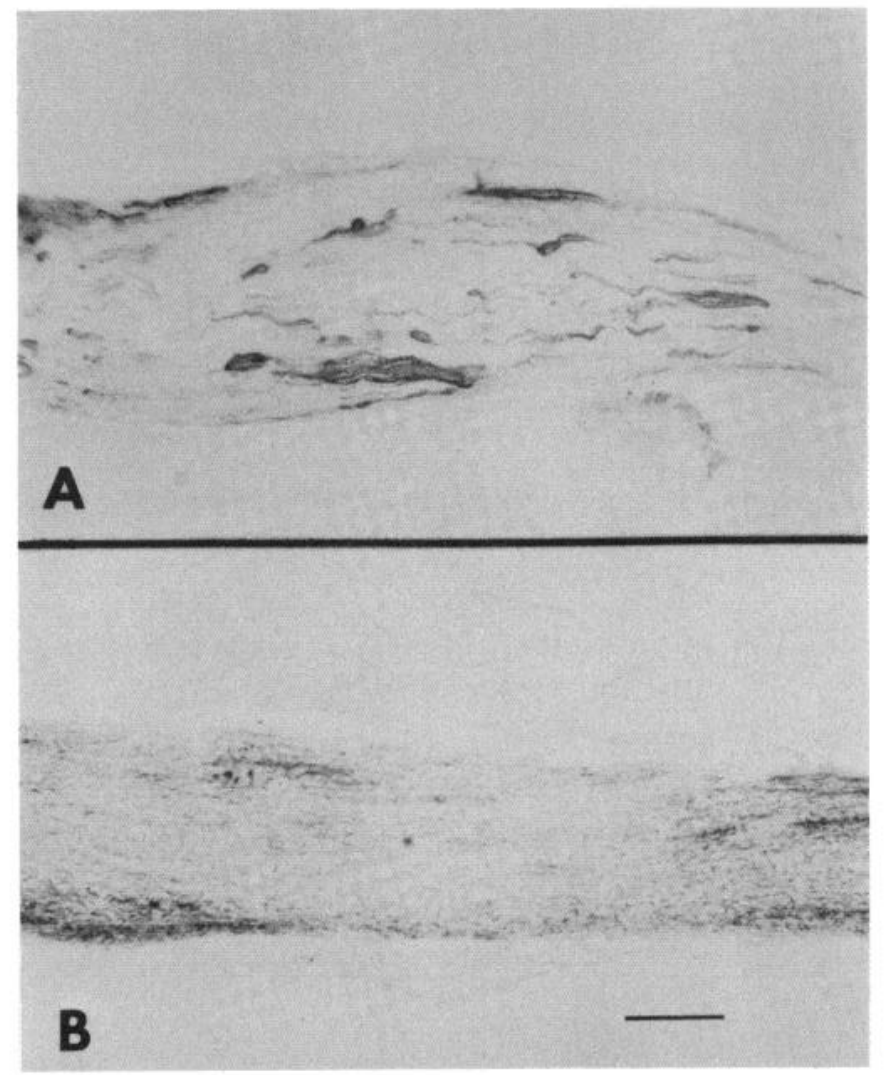

Figure 4. Staining for cholinesterase in the lingual-chorda tympani nerve. $A$, Twenty-four hours of exposure to a colchicine cuff caused cholinesterase accumulation in many axons. $B$, Contralateral control nerve. Scale line, $0.1 \mathrm{~mm}$. 
Nerve ultrastructure. In nerves treated with colchicine cuffs for 3 days, several structural changes were observed which were not seen in nerves having control cuffs for 3 days. These changes, observed immediately distal to the cuff site, were more pronounced in 8- and 15-day cuffed nerves. They include $(i)$ more prominent and disoriented neurofilaments, (ii) a reduction in the number of microtubules, (iii) accumulations of clusters of mitochondria, (iv) thinning of the myelin sheath, and $(v)$ loss of axoplasm. We used a computer graphics tablet to document the thinning of the myelin and the loss of axoplasm which was evident as a retraction of the axolemma from the innermost lamella of myelin. The effects of colchicine upon nerve ultrastructure are described more fully in a separate communication (S. E. Hughes, H. E. Sloan, L. B. Jones, and B. Oakley, submitted for publication).

\section{Discussion}

Our observations are consistent with previous reports that colchicine treatment causes taste bud degeneration (Beidler and Smallman, 1965; Chelyshev et al., 1981; Donegani and Filogamo, 1971; Rodrigo Angulo et al., 1978) and a loss of summated taste responses from the chorda tympani nerve (Beidler and Smallman, 1965). The central objective of our research has been to determine whether the chronic disruption of axonal transport by colchicine is sufficient to produce a loss of taste responses and taste buds. Colchicine has several actions in addition to blocking axonal transport. Consequently, it seems necessary to rule out these actions before axonal transport can be implicated in the neurotrophic maintenance of taste buds.

A small nerve cuff proved to be an effective device for restricting colchicine to the lingual-chorda tympani nerve. In gerbils in which taste responses disappeared after 3 to 4 days, the impulse traffic of compound action potentials continued to propagate along the nerve and through the cuffed region.

It is unlikely that the systemic distribution of colchicine from Silastic cuffs on the lingual-chorda tympani nerve caused taste bud dysfunction. Taste responses were unaffected by inferior alveolar nerve cuffs containing up to 50 times more colchicine. Moreover, measurements utilizing $\left[{ }^{3} \mathrm{H}\right]$ colchicine argue against direct actions of colchicine on the taste buds whatever the route of entry to the tongue. These measurements showed that for at least the first $48 \mathrm{hr}$ of lingual-chorda tympani nerve treatment the colchicine concentrations were virtually identical on the two sides of the tongue (Fig. 3). Since the fungiform taste buds on the contralateral side of the tongue remained morphologically and physiologically normal, the site of direct action of colchicine must have been at the nerve trunk and not at the taste buds of the tongue.

Conceivably, colchicine might have disrupted nerve trunk functions unrelated to axonal transport but critical for the maintenance of the taste system. However, cuffs containing lumicolchicine failed to impair taste function. Lumicolchicine is an isomer of colchicine that, like colchicine, binds to membranes (Stadler and Franke, 1974) and inhibits nucleoside uptake (Mizel and Wilson, 1972). Unlike colchicine, it does not block axonal transport (Banks and Till, 1975; Heiwall et al., 1978) and only weakly binds to the protein tubulin that is strongly implicated in axonal transport (Borisy et al., 1972).

The accumulation of axonal cholinesterase and mitochondria indicated that colchicine, when applied by the present cuff method, actually impaired axonal transport as predicted from prior demonstrations of the blocking action of colchicine on axonal transport (Dahlström, 1968; Fink et al., 1973; Kreutzberg, 1969; Sjöstrand et al., 1970). If proximodistal axonal flow maintains axoplasm volume, as Hansson and Sjöstrand (1971) suggest, then the observed reduction in axoplasm distal to the cuff represents additional evidence of transport blockage by colchicine. The use of blank control cuffs demonstrated that the mechanical irritation of the cuff technique produced significantly less impairment of taste responses and taste buds than did colchicine. In light of the above evidence against alternative modes of action, it seems likely that nerve cuffs eliminated taste responses and taste buds by the specific binding of colchicine to tubulin that disrupted axonal transport in the lingual-chorda tympani nerve. The proximate objective of many studies which treat axons with colchicine is to block axonal transport without degrading the structure and function of the nerve trunk. Over the long term this may be an unsustainable objective since axons themselves undoubtedly depend upon materials transported to and from the neuron cell body. Nonetheless, we found it possible in the present work, as have others using similar assays (Jackson and Diamond, 1977), to select a treatment regimen which would promptly impair axonal transport while leaving the compound action potential functional and the nerve without degenerated axons.

Colchicine produced a nearly complete loss of taste responses between 3 and 8 days and of fungiform taste buds between 8 and 15 days. Such temporally dispersed actions upon structure and function are unlikely to have resulted from variable access of colchicine to the chorda tympani axons since the HRP demonstrated that in the vicinity of the nerve cuff the chorda tympani axons remained in a single tight bundle $(<100 \mu \mathrm{m}$ in diameter) on the perimeter of the lingual nerve. Hence, the chorda tympani axons probably were exposed to colchicine simultaneously. Yet, we observed that adjacent axons often differed substantially in the degree of ultrastructural disruption. This suggests that the temporal dispersion of the taste response and taste bud losses may reflect the variable reaction of axons to colchicine and of taste buds to a reduction in neurotrophic substances.

We chose to examine the gerbil's chorda tympani nerve because its accessibility in the middle ear made the recording of taste responses technically straightforward. In previous acute experiments, the chorda tympani taste responses from the rat or gerbil have been observed to decline after nerve transection with a latency of 6 to 12 $\mathrm{hr}$ and to disappear after about $24 \mathrm{hr}$ (Hellekant et al., 1979; Oakley et al., 1979). In the present study the onset of the taste response decline occurred more than 1 day after implantation of colchicine cuffs. This longer latency may, in part, be a consequence of the time required for colchicine to diffuse from the Silastic cuff and to penetrate the nerve sheath and myelinated axons in adequate concentrations (Fink et al., 1973).

Depletion of slowly transported materials cannot ac- 
count for the reduced taste responses observed here. Fifty percent of the fungiform taste buds are found within $2.7 \mathrm{~mm}$ of the tip of the gerbil tongue, 18 to $21 \mathrm{~mm}$ distal to the nerve cuff site (Cheal and Oakley, 1977). Assuming continued transport distal to the cuff and a rough proportionality between the magnitude of the taste response and the number of responsive fungiform taste buds (Miller, 1976), at a transport velocity of $4 \mathrm{~mm} /$ day it would have required at least 4 days to deprive the posterior $50 \%$ of the fungiform taste buds of transported materials. Yet, 3 days after applying a cuff containing colchicine, the taste responses already were reduced by more than $60 \%$. Thus, the substances required for continued gustatory function must be transported at a rate exceeding $4 \mathrm{~mm} /$ day. This is a conservative estimate of a lower bound for the transport rate since it takes into account neither the latency for colchicine to disrupt transport nor the possibility of a reservoir of trophic agents sequestered at the axon terminal or receptor cell. A requirement for transport exceeding $4 \mathrm{~mm} /$ day eliminates several slowly transported substances and organelles as possible trophic agents (Grafstein and Forman, 1980).

The taste buds are the classic example of a trophic system in which the end organ depends upon normal innervation. In contrast to skeletal muscle, it is unlikely that impulse-associated activity in taste buds is essential for re-forming or maintaining the end organ because (i) regenerating taste fibers have little or no spontaneous activity until after taste buds regenerate (Cheal et al., 1977), (ii) sensory impulses are directed away from the taste bud, and (iii) de-efferentation by decentralization of the sensory ganglia does not cause the loss of taste responses or taste buds (Berland et al., 1977; Donegani and Gabella, 1967; Donoso and Zapata, 1976). Alternatively, the neurotrophic maintenance of taste buds may require axonal transport of chemical substances along taste axons. This could involve, for example, the release of a neurohumor, as proposed by Olmsted in 1920, or the removal of an inhibitory factor. Recently, it has been shown that acute disruptions of the gerbil IXth nerve by local cooling, by colchicine, or by transection at different lengths trigger a decline of taste discharges attributable to impaired axonal transport to the taste buds (Oakley et al., 1979, 1980, 1981). In the present study we used colchicine-containing Silastic nerve cuffs to determine whether axonal transport also is required to maintain taste bud structure. The results of these experiments indicate that colchicine produced a loss of taste buds and taste responses by disrupting axonal transport in the lingual-chorda tympani nerve and not by other toxic actions to the nerve or to cells of the taste bud. From this work and earlier studies the sequence of events following cholchicine treatment secms to be: $(i)$ impairment of axonal transport, (ii) failure of taste responses, (iii) taste bud degeneration, and ultimately with continued action of colchicine, (iv) a loss of the compound action potential and nerve degeneration. Although this sequence of events certainly is compatible with the view that taste axon endings release diffusible neurotrophic agents which act upon epithelial cells in the tongue, considerable further evidence will be necessary to estab- lish the existence and exact mode(s) of action of axonally transported substances required to maintain taste buds.

\section{References}

Banks, P., and R. Till (1975) A correlation between the effects of anti-mitotic drugs on microtubule assembly in vitro and the inhibition of axonal transport in noradrenergic neurones. J. Physiol. (Lond.) 252: 283-294.

Beidler, L. M., and R. L. Smallman (1965) Renewal of cells within taste buds. J. Cell Biol. 27: 263-272.

Berland, D. W., J. S. Chu, M. A. Hosley, L. B. Jones, J. M. Kaliszewski, W. C. Lawler, and B. Oakley (1977) New approaches to the problem of the trophic function of neurons. In Olfaction and Taste VI, J. LeMagnen and P. MacLeod, eds., pp. 217-224, Information Retrieval Ltd., London.

Borisy, G. G., J. B. Olmstead, and R. A. Klugman (1972) In vitro aggregation of cytoplasmic microtubule subunits. Proc. Natl. Acad. Sci. U. S. A. 69: 2890-2894.

Cheal, M., and B. Oakley (1977) Regeneration of fungiform taste buds: Temporal and spatial characterizations. J. Comp. Neurol. 172: 609-626.

Cheal, M., W. P. Dickey, L. B. Jones, and B. Oakley (1977) Taste fiber responses during reinnervation of fungiform papillae. J.Comp. Neurol. 172: 627-646.

Chelyshev, Y. A., T. L. Zefirov, and V. M. Ivanov (1981) Tongue taste buds after colchicine applications to the rat glossopharyngeal nerve. Bull. Exp. Biol. Med. 91: 572-574.

Colman, D. R., F. Scalia, and E. Cabrales (1976) Light and electron microscopic observations on the anterograde transport of horseradish peroxidase in the optic pathway in the mouse and rat. Brain Res. 102: 156-163.

Conger, A. D., and M. A. Wells (1969) Radiation and aging effect on taste structure and function. Radiat. Res. 37: 31-49.

Dahlström, A. (1968) Effect of colchicine on transport of amine storage granules in sympathetic nerves of rat. Eur. J. Pharmacol. 5: 111-113.

Donegani, G., and G. Filogamo (1971) Sul comportamento dei recettori nelle papille foliate dopo trattamento con colchicina del nervo glosso-faringeo, nel coniglio. Boll. Soc. Ital. Biol. Sper. 47: 156-159.

Donegani, G., and G. Gabella (1967) Effecto della sezione intracrancia del nervo glosso-faringeo sui corpuscoli gustativi, nel coniglio. Boll. Soc. Ital. Biol. Sper. 43: 1165-1167.

Donoso, A., and P. Zapata (1976) Effects of denervation and decentralization upon taste buds. Experientia 32: 591-592.

Farbman, A. I. (1980) Renewal of taste bud cells in circumvallate papillae. Cell Tissue Kinet. 13: 349-357.

Fink, B. R., M. R. Byers, and M. E. Middaugh (1973) Dynamics of colchicine effects on rapid transport and axonal morphology. Brain Res. 56: 299-311.

Fujimoto, S., and R. G. Murray (1970) Fine structure of degeneration and regeneration in denervated rabbit vallate taste buds. Anat. Rec. 168: 398-413.

Goshgarian, H. G. (1977) A rapid silver impregnation for central and peripheral nerve fibers in paraffin and frozen sections. Exp. Neurol. 57: 296-301.

Grafstein, B., and D. S. Forman (1980) Intracellular transport in neurons. Physiol. Rev. 60: 1167-1283.

Guth, L. (1957) The effects of glossopharyngeal nerve transection on the circumvallate papilla of the rat. Anat. Rec. 128: 715-731.

Hansson, H. -A. and J. Sjöstrand (1971) Ultrastructural effects of colchicine on the hypoglossal and dorsal vagal neurons of the rabbit. Brain Res. 35: 379-396.

Heiwall, P. -O., P. -A. Larsson, and A. Dahlström (1978) Further evidence for the involvement of microtubules in the proximo- 
distal intra-axunal transport of acetylcholine and related enzymes in the rat sciatic nerve. Acta Physiol. Scand. 104: 156-166.

Hellekant, G., V. Gopal, and Y. Ninomiya (1979) Decline and disappearance of taste response after interruption of the chorda tympani proper nerve of the rat. Acta Physiol. Scand. 105: 52-57.

Jackson, P., and J. Diamond (1977) Colchicine block of cholinesterase transport in rabbit sensory nerves without interference with the long-term viability of the axons. Brain Res. 130: 579-584.

Jakinovich, W., Jr., and B. Oakley (1975) Comparative gustatory responses in four species of gerbilline rodents. J. Comp. Physiol. 99: 89-101.

Kreutzberg, G. W. (1969) Neuronal dynamics and axonal flow. IV. Blockade of intra-axonal enzyme transport by colchicine. Proc. Natl. Acad. Sci. U. S. A. 62: 722-728.

Miller, I. (1976) Taste bud distribution and regional responsiveness on the anterior tongue of the rat. Physiol. Behav. 16: 439-444.

Mizel, S. B., and L. Wilson (1972) Nucleoside transport in mammalian cells. Inhibition by colchicine. Biochemistry 11 : 2573-2577.

Oakley, B., L. B. Jones, and M. A. Hosley (1979) Decline of IXth nerve taste responses following nerve transection. Chem. Senses Flav. 4: 287-299.

Oakley, B., L. B. Jones, and M. A. Hosley (1980) The effect of nerve stump length upon mammalian taste responses. Brain Res. 194: 213-218.
Oakley, B., J. S. Chu, and L. B. Jones (1981) Axonal transport maintains taste responses. Brain Res. 221: 289-298.

Olmsted, J. M. D. (1920) The nerve as a formative influence in the development of taste buds. J. Comp. Neurol. 31: 465-468.

Rodrigo Angulo, M. L., B. Fernández Sánchez, and E. L. Rodríguez-Echandía (1978) The reversible effect of colchicine on the taste bud cells of the central circumvallate papilla in the rat. Cell Tissue Res. 192: 67-76.

Sjöstrand, J., M. Fizell, and P. -O. Hasselgren (1970) Effects of colchicine on axonal transport in peripheral nerves. J. Neurochem. 17: 1563-1570.

Stadler, J., and W. W. Franke (1974) Characterization of the colchicine binding of membrane fractions from rat and mouse liver. J. Cell Biol. 60: 297-303.

State, F. A., and H. I. Dessouky (1977) Effect of the length of the distal stump of transected nerve upon the rate of degeneration of taste buds. Acta Anat. (Basel) 98: 353-360.

State, F. A., M. S. Hamed, and A. A. Bondok (1977) Effect of vincristine on the histological structure of taste buds. Acta Anat. (Basel) 99: 445-449.

Venable, J. H., and R. Coggeshall (1965) A simplified lead citrate stain for use in electron microscopy. J. Cell Biol. 25: 407-408.

Vintschgau, M. von, and J. Hönigschmied (1877) Nervus Glossopharyngeus und Schmeckbecher. Arch. Gesamte Physiol. 14: 443-448.

Wilson, L., and M. Friedkin (1966) The biochemical events of mitosis. I. Synthesis and properties of colchicine labeled with tritium in its acetyl moiety. Biochemistry 5: 2463-2468. 